\title{
Context Awareness for Self-adaptive and Highly Available Production Systems
}

\author{
Sebastian Scholze ${ }^{1}$, José Barata ${ }^{2}$, and Oliver Kotte ${ }^{1}$ \\ ${ }^{1}$ Institut für angewandte Systemtechnik Bremen GmbH, 28359 Bremen, Germany \\ \{scholze, kotte\} aatb-bremen. de \\ ${ }^{2}$ CTS - UNINOVA, Dep. De Eng. Electrotecnica, Faculdade de Ciencias e Tecnologica, \\ Universidade Nova de Lisboa, 2829-516 Caparica, Portugal \\ jab@uninova.pt
}

\begin{abstract}
A new approach for the realization of self-adaptive and highly available production systems based on a context aware approach, allowing self-adaptation of flexible manufacturing processes in production systems and effective knowledge sharing to support maintenance, is presented. The usage of dynamically changing context as basis for adaptation of flexible manufacturing lines/processes and effective knowledge sharing is proposed. The presented solution includes services for context extraction, adaptation and self-learning allowing high adaptation of production systems depending on the identified context. A generic architecture following Service Oriented Principles is presented allowing for integration of the proposed solution into various production systems. A successful initial application of the developed solution in real world manufacturing environment is presented.
\end{abstract}

Keywords: Context Awareness, Context Extraction, Ontology, SOA.

\section{Introduction}

The need for highly flexible manufacturing processes is nowadays indispensable. In order to enhance the availability and at the same time the efficiency of modern production systems, two key requirements arise a) self-adaptation to support change of process parameters aiming at increasing flexibility and efficiency and b) effective knowledge acquisition / sharing to support maintenance increasing availability of production systems, as indicated in [1].

In order to realize such production systems, which are able to solve both above mentioned requirements, there is a clear need for powerful ICT infrastructures/solutions able to handle high amount of data, complex models and algorithms. Key challenge is to find a common approach for addressing these two problems:

- To allow for a self-adaptation, production systems need advanced monitoring and control. Thereby changes (e.g. changing process parameters) need to be identified and used to (self) adapt control processes. 
- To allow for knowledge sharing, production systems need advanced monitoring to allow enhancement of monitored knowledge in order to facilitate knowledge sharing needed for effective maintenance.

A service oriented approach promises new perspectives for realizing such selfadaptive production systems. It is likely that approaches based on Service Oriented Architecture (SOA) principles, using distributed networked embedded services in device space (sensors, controllers etc.), are the most appropriate for implementation of such production systems in general.

While successful application of SOA based principles in manufacturing lines is emerging, there is still a major challenge:

How to realize reliable, production systems for complex manufacturing processes, which support both, self-adaption of process parameters and knowledge sharing to support improved maintenance of such production systems, while using a common approach.

\subsection{Research Question}

Taking the above mentioned major challenge and the related sub-challenges into account, a new innovative approach for future production systems is required, which raises the following research question:

Which methods and tools are required, to allow for the realization of reliable production systems that are able to support both, self-adaption of production processes without the need to reprogram or reconfigure such manufacturing processes and knowledge sharing to support effective maintenance, increasing thereby availability and efficiency of complex manufacturing processes?

The research question elaborated above can be addressed by the following two hypotheses:

- Self-adaptive production systems can be achieved if a context aware approach is used to identify the current context of (complex) manufacturing processes and use this extracted context as a basis for self-adaptation of process parameters.

- Effective sharing of knowledge in production systems can be achieved, if a context aware approach is used to identify the current context under which the knowledge is monitored and use this extracted context as foundation to support knowledge sharing, improving effective maintenance.

\section{Relationship to Internet of Things}

The application of context awareness within production systems to allow the realization of self-adaptive and highly available systems may on one side lead to enormous benefits regarding availability and efficiency of production systems and, on the other side increase the implementation of such possibilities by using a generic solution which can be used in several scenarios (i.e. Control, Maintenance). 
To allow for the realization of such solutions, the presented work describes a new approach for such self-adaptive production systems based on a context aware approach, allowing self-adaptation of flexible manufacturing processes in production systems. The usage of dynamically changing context of production lines, as well as changing ambient conditions, for adaptation of flexible manufacturing lines/processes based on the usage of connected sensors and machines, as well as scalable architectures is proposed.

\section{Survey of the State of the Art}

This section gives a brief overview of the state-of-the-art for RTD topics which are relevant for the presented approach.

In the area of self-learning production systems, the research has demonstrated that the application of machine learning techniques, dynamic self-adaptation and operator's feedback in the loop promises to increase the intelligence of the overall system. In this approach, machine learns whenever it changes its structure, program, or data based on inputs or in response to external information in such a manner that its overall performance is expected to improve [2], [3], [4]. In production systems in particular, these methods have been proven to be especially useful for monitoring/diagnosis [5], [6], [7] and control [8]. However, the applications of self-adaptation of production systems and learning in industrial practice are still in initial phase. In this paper a novel approach in production systems is presented, which is context aware, adaptive to contextual changes at run time and learns from adaptation and operator's action.

Of high interest for the work presented in this paper is Service Oriented Architecture (SOA) in manufacturing i.e. the relation between self-learning production systems and SOA. SOA gained popularity with the advancement of web service technologies in industrial networks. For example, Devices Profile for Web Services (DPWS) allows devices with embedding computing capabilities, sensors, actuators etc. to interact within the industrial network and enabling SOA integration to higher levels [9]. OPC Unified Architecture is the recent OLE (Object Linking and Embedding) for process control (OPC) specification from the OPC Foundation and differs significantly from its predecessors. The Foundation's goal was to provide a path forward from the original OPC communications model (namely COM/DCOM) to a cross-platform SOA for process control, while enhancing security and providing an information model [10]. Scalable SOA holds promise for seamless integration, interoperation and flexibility in manufacturing environment. But, there is a lack of adoption of overall SOA based self-adaptive production systems in discrete manufacturing environment.

Context awareness is widely applied in modern ICT solutions. Different approaches to context modeling are developed, where ontology based context modeling is mostly investigated. Based on the formal description of context information, context can be processed with contextual reasoning mechanisms. Since contextual information has some inherent features (it can be considered incomplete, temporal, and interrelated) context reasoning can exploit reasoning mechanisms to deduce high level, inferred context from low-level raw contextual information. Furthermore, contextual 
reasoning can be used to verify and possibly solve inconsistent context knowledge due to imperfect input. For example, Luther et al. [11] show the needs for ontology support and reasoning in mobile applications; their case study is conducted with the Protégé knowledge workbench [12] for ontology modeling and OWL editing, and the RACER inference engine [13] for proof checking, ontology validation and classification. A more flexible use of ontological reasoning is presented in [26]. Their framework utilizes context-awareness for service classification.

Application of context awareness for SOA based self-learning production systems has not yet been sufficiently investigated. The approach proposed in this paper applies the ontology based context modeling, and re-use of experience of other projects [14], for context extraction in highly flexible and dynamic self-learning production systems [15].

\section{Proposed Approach to Identify Context}

The key assumption is that the extraction of current context is the foundation for selfadaptation / self-configuration of service-oriented production systems on one side, as well as for knowledge sharing to support effective maintenance. The application of such a context aware approach based on extraction of dynamically changing current context seems to be an effective way to assure availability and efficiency of modern, flexible production systems.

In a more detail, the assumption is that it is possible to gain more efficient embedded services by using context awareness than by using classical embedded services. The foundation of such context aware services is data, which will be gathered from various sources (e.g. sensors, inputs of the human operator, etc.). This information will be used to identify the current context of the services, which is realized via monitoring services, which are, for example, services for monitoring of sensors or of a user interacting with a system. These Monitoring services will transform all monitored information into a "standardized" matrix/action which is used to extract the current context. The extraction uses the Monitoring/Action Matrix together with context reasoning methods to identify the context. Basis of the context identification is a context model for device spaces. The context model consists of a generic model, to allow for effective use of different application domains, and a specific model which instantiates generic concepts to allow for optimal adjustment to the domain. In order to allow context extraction to produce tangible results, a well-defined context model is crucial.

The identified context will be used to a) allow embedded services to adopt their behavior according to the identified context (e.g. change of process parameters) and/or b) support sharing of knowledge of production processes according to the identified context (e.g. identify necessary maintenance tasks).

The objective of the presented work is to realize a context aware approach which can be used as a common solution for the realization of self-adaptive production systems and effective knowledge sharing to support effective maintenance in such production systems. The aim is to develop a generic solution, which can be used in several scenarios (i.e. Control, Maintenance) and various domains [16], [17], [18]. 


\subsection{Architecture}

A context aware approach allows a manufacturing system to be dynamically self-adapted during run time. To achieve this, the solution adapts based on contextual changes and learns from these adaptions. The learning is further enhanced by the operator's feedback.

A generic solution for context based self-adaptation of production systems is proposed which can be applied in various manufacturing systems. This self-learning reference architecture is derived from analyzing three application cases which belong to different industrial sectors. The overall proposed reference architecture, which is following SOA principles, is illustrated in Figure 1.

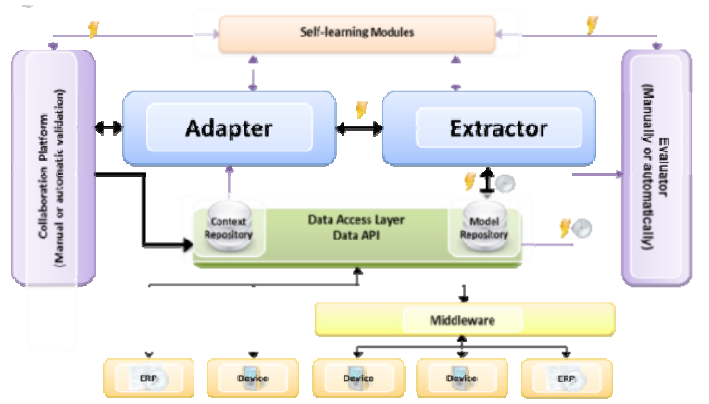

Fig. 1. Proposed Reference Architecture [19],[20]

The modules of the reference architecture are not described in this paper, for a detailed description see also [21].

\subsection{Context Extraction}

Context Extraction is based on a set of embedded services responsible for identifying changes in the context of the environment (see Figure 2). The current identified context is used to extract available context knowledge. The results of the Context Extraction are used in the Adapter which is responsible for updating the system behavior.

Context Extraction uses all "raw data" provided via the data access layer to derive the machine's current contextual situation [22]. Using the ontology/context model the monitored data is evaluated and the current context extracted [23]. Based on the identified context, situations can be compared to previous ones and stored. A continuous process, which is coordinating with the monitoring and followed by the adaption process to give current contextual meaning to the provided knowledge, is built around the main extraction of current contextual. The core modules of the proposed Context Extractor Architecture are the following:

- Adapter Interface: Via this interface the Context Extractor and the Adapter will exchange data and call specific functionality on both sides.

- Data Access Layer: Responsible for accessing the device layer. 
- Data Processing: This module is responsible for the pre-processing of monitored raw data acquired via the data access layer, before the context will be identified. Main functionality will be the normalization of monitored data to transform the raw data in a format which serves as basis for context identification

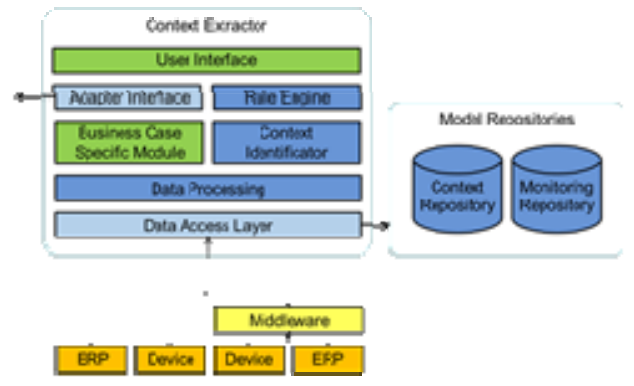

Fig. 2. Context Extractor Architecture

- Context Identifier: Main component of the Context Extractor. It is responsible for the identification of the current context, based on monitored raw data, the ontology and historic context information stored in the context repository.

- Rule Engine: Responsible for providing rules for the identification of context.

- User Interfaces: User interfaces for maintaining and administering the rules and the context repository.

- Model Repositories: Inside the Context Repository the identified context will be stored for further processing and reuse. Model Repository is used for the ontology.

- Business Case Specific Modules: This module is a placeholder and represents all components and user interfaces which needs to be developed for each business case individually.

\section{Application}

The presented concept has been developed following SOA principles and initially applied in different application scenarios. These application scenarios belong to different industrial sectors.

One of these application scenarios addresses control systems/machines and automation systems for shoe industry. The overall objective is to enhance machines with selfadaptive functionalities by allowing machines to inspect statistically the condition of products and equipment, report and analyze proactively the gathered statistic values, enabling the machines to decide and adapt the parameters and keep them always inside the "optimized" working range. The objective is to achieve high adaptation of the machines to the changing conditions. The parameter variations are in terms of pressure and temperature, speed frequencies of drives and pumps, proper material mix ratio and filling of materials into shoe forms. For example, one of the scenarios addresses synchronicity of the different valve circuits when dosing of several components, caused by e.g. 
different force requirements or different air supply. As the valve synchronization is designed by an electronic system with different valve opening times, and/or due to valve abrasion, it may come to flaws in the product. Currently the synchronization can only be adjusted by a technician during downtime of the machines. By implementation of the proposed self-adaptive solution an automatic adjustment of the valve switching to different conditions is achieved. The advantage of having such an intelligent solution for the valve synchronizations is that it provides a basis for preventive maintenance.

The implementation of the proposed self-adaptive solution for the automatic adjustment of machine parameters based on changing context, for example changing ambient conditions, leads to minimization of errors and keeps the machine utilization high, as well as the overall product quality. The context extraction serves as a basis for identifying machine adjustment parameters.

\section{Conclusions and Further Work}

A novel approach for the realization of self-adaptive and highly available production systems is presented. The proposed solution addresses the adaptation of process parameters based on a context aware approach.

Further research will focus on advanced algorithms for self-learning based on extracted context to (semi-)automatically update the context model. In addition the context model itself will be addressed by further research to allow better utilization of the presented model for other companies as well as for other application domains. High complexity of data acquisition and real-time data analysis algorithms will be addressed in further research to "fully" utilize the opportunities offered by service-based self-learning systems.

Acknowledgements. This work is partly supported by the Self-Learning project of European Union's 7th Framework Program, under the grant agreement no. NMP-2008228857 and by the EPES project of European Union's 7th Framework Program, under the grant agreement no. FoF-ICT-2011.7.3-285093. This document does not represent the opinion of the European Community, and the European Community is not responsible for any use that might be made of its content.

\section{References}

1. MANUFUTURE Strategic Research Agenda. Report of the High-Level Group (September 2006)

2. Alpaydin, E.: Introduction to machine learning. The MIT Press (2004)

3. Michie, D., Spiegelhalter, D., Taylor, C., Campbell, J.: Machine learning, neural and statistical classification. Ellis Horwood Series in Artificial Intelligence, p. 289 (1995)

4. Carbonell, J., Michalski, R., Mitchell, T.: An overview of machine learning. Machine Learning: An Artificial Intelligence Approach 1, 3-23 (1983)

5. Liu, S.C., Liu, S.Y.: An Efficient Expert System for Machine Fault Diagnosis. The International Journal of Advanced Manufacturing Technology 21, 691-698 (2003) 
6. Palluat, N., Racoceanu, D., Zerhouni, N.: A neuro-fuzzy monitoring system application to flexible production systems. Comput. Ind. 57, 528-538 (2006)

7. Jianbo, Y., Lifeng, X., Xiaojun, Z.: Intelligent monitoring and diagnosis of manufacturing processes using an integrated approach of KBANN and GA. Comput. Ind. 59, 489-501 (2008)

8. Monostori, L.: AI and machine learning techniques for managing complexity, changes and uncertainties in manufacturing. Engineering Applications of Artificial Intelligence 16, 277-291 (2003)

9. Ribeiro, L., Barata, J., Colombo, A.W., Jammes, G.: A Generic Communication Interface for DPWS-based Web Services. In: IEEE International Conference on Industrial Informatics, INDIN, Daejeon, Korea. IEEE (2008)

10. Mahnke, W., Leitner, S.-H.: OPC Unified Architecture - The future standard for communication and information modeling in automation. ABB Review 3/2009, pp. 56-61 (2009)

11. Luther, M., et al.: Situational reasoning - a practical OWL use case, Chengdu, Jiuzhaigou, China. IEEE (2005)

12. Noy, F.N., et al.: Creating Semantic Web contents with Protege-2000. IEEE Intelligent Systems 16(2), 60 (2000)

13. Haarslev, V., Moller, R.: RACER system description, Siena, Italy. Springer (2001)

14. Ziplies, S., Scholze, S., Stokic, D., Krone, K.: Service-based Knowledge Monitoring of Collaborative Environments for User-context Sensitive Enhancement. In: ICE 2009 (2009)

15. Sorli, M., Stokic, D.: Innovating in Product/Process Development. Springer, Heidelberg (2009)

16. K-NET Project - Deliverable D1.4 Public Concept (2008)

17. AsKoWi Project (2011), http: / /www . askowi.de

18. EPES Project (2011), http: / / www . epes-project. eu

19. Self-Learning, EU project NMP-2008-228857 "Reliable self-learning production system based on context aware services", Public report (2010)

20. Stokic, D., Scholze, S., Barata, J.: Self-Learning Embedded Services for Integration of Complex, Flexible Production Systems. In: IECON 2011-37th Annual Conference on IEEE Industrial Electronics Society, Melboure, Australia (2011)

21. Scholze, S., Stokic, D., Barata, J., Decker, C.: Context extraction for self-learning production systems. In: 2012 10th IEEE International Conference on Industrial Informatics (INDIN), July 25-27, pp. 809-814 (2012)

22. Kelly, D., Teevan, J.: Implicit Feedback for Inferring User Preferences: A Bibliography. SIGIR Forum 37(2), 18-28 (2003)

23. Oard, D.W., Kim, J.: Modelling information content using observable behaviour. In: Proc. ASIST Annual Meeting, pp. 481-488 (2001) 